\title{
Active Galactic Nuclei and Transformation of Dark Matter into Visible Matter ${ }^{1}$
}

\author{
A. A. Grib ${ }^{2,3^{*}}$ and Yu. V. Pavlov ${ }^{2,4^{* *}}$ \\ ${ }^{2}$ A. Friedmann Laboratory for Theoretical Physics, \\ Griboedov kanal 30/32, St. Petersburg 191023, Russia \\ ${ }^{3}$ Russian State Pedagogical University (The Herzen University), \\ Moyka-river Emb. 48, St. Petersburg 191186, Russia \\ ${ }^{4}$ Institute of Mechanical Engineering, Russian Acad. Sci., \\ Bolshoy pr. 61, St. Petersburg 199178, Russia
}

\begin{abstract}
The hypothesis that dark matter is converted into visible particles in active galactic nuclei is investigated. If dark matter consists of stable superheavy neutral particles and active galactic nuclei are rotating black holes, then, due to the Penrose process, superheavy particles can decay into unstable particles with larger mass, whose decay into quarks and leptons leads to events in cosmic rays observed by the Auger group. Similar processes of decay of superheavy particles of dark matter into visible matter occurred in the early Universe. Numerical estimates of the processes in active galactic nuclei and in the early Universe are given.
\end{abstract}

PACS numbers: 98.80.Cq, 95.35.+d, 98.70.Sa

\section{INTRODUCTION}

Recently the Auger group for observation of cosmic rays claimed [1] that 27 events with ultra high energy $E \geq 57 \times 10^{18} \mathrm{eV}$ were observed. Some correlation of these ultra high energy cosmic rays (UHECR) with the distribution of nearest active galactic nuclei (AGN) was also observed.

In our previous papers [2] we tried to explain these events by the hypothesis investigated by us [3] and some other authors 4 that dark matter consists mainly of neutral stable superheavy particles with masses of the order of the Grand Unification (GU) scale $M_{U}=10^{14}-10^{15} \mathrm{GeV}$. The argument for this was due to numerical estimate obtained earlier [5, 6] that gravitation of the early expanding Universe could produce the observable number of visible particles (the Eddington number) if it first created from vacuum superheavy particles which then decayed into visible ones but part of them survived till today as dark matter.

Supposing that similar to $K^{0}$-meson theory, superheavy particles can exist in two forms, the long-

\footnotetext{
*E-mail: andrei_grib@mail.ru

**E-mail: yuri.pavlov@mail.ru

${ }^{1}$ Plenary talk given at the International Conference RUSGRAV-13, June 23-28, 2008, PFUR, Moscow
}

living component existing today and the short-living one, which decayed in the early Universe, it is interesting to investigate the idea that in AGN the process of converting long-living components into shortliving ones, decaying into visible particles, can occur. As it was said in [2], this can be the Penrose process in rotating black holes [7]. As it is known, in this process the superheavy particle in ergosphere of the rotating black hole can decay into two particles: one with the negative energy, the other with positive energy. So it is possible that the particle with positive energy can have a larger mass than the decaying one. Calling these particles $X_{l}$ and $X_{s}$, one can have $m_{X_{l}}<m_{X_{s}}$. In this sense, such situation is different from the usual $K^{0}$-meson theory with $m_{K_{l}}>m_{K_{s}}$. Then $X_{s}$ decays into quarks and leptons of superhigh energies. The flow of these particles escapes the AGN, and some of them come to the Earth.

It is clear that in ordinary space, as well as in galactic nuclei which are not rotating black holes, this process cannot be observed and dark matter is stable.

The structure of the paper is the following. First we analyze the Penrose process in AGN as rotating black holes. Then we repeat our arguments on the role of superheavy particles in the early Universe. 


\section{Superheavy particles as sources of UHECR from active galaxy nuclei}

Let us give some numerical estimates of the conversion of superheavy particles into UHECR in active galactic nuclei. The Auger group detected 27 UHECR with energies higher than $57 \times 10^{18} \mathrm{eV}$. The integrated exposure of the Auger observatory for these data is $9.0 \times 10^{3} \mathrm{~km}^{2}$ sr year. The Auger group found the correlation of UHECR with nearby active extragalactic objects [1]. There are 318 AGN in the field of view of the Observatory at distances smaller than $75 \mathrm{Mpc}$. It is easy to see that if these AGN are distributed uniformly and have the same intensity of UHECR radiation, each of the AGN must radiate approximately $j=10^{39}$ UHECR per year. The distance of propagation of UHECR is limited by the Greizen-Zatsepin-Kuzmin limit 8 and for a proton with an energy of the order $8 \times 10^{19} \mathrm{eV}$ this distance cannot be larger than $90 \mathrm{Mpc}$.

Due to the Auger results, the source of 2 particles of superhigh energy is the AGN Centaurus A located at 11 million of light years from the Earth. It is easy to calculate that for the integral exposure of the Auger observatory this AGN must radiate $j \approx 3 \times$ $10^{37}$ UHECR per year.

There is no well established theory explaining the origin of cosmic particles with energies higher than $10^{18} \mathrm{eV}$ in AGN. Our hypothesis is that these UHECR in AGN arise due to superheavy dark matter particles converted into quarks and leptons at high energies obtained by them close to the supermassive black hole horizon of the AGN. Superheavy dark matter particles with a mass $M=10^{14} \mathrm{GeV}$ $\approx 2 \times 10^{-10} \mathrm{~g}$, fall onto the black hole, so that if $100 \%$ of these particles are converted into UHECR, then it's mass must have the order of $M j \sim 10^{28} \mathrm{~g}$. Even if only $\eta=10^{-4}$ of the total mass of superheavy particles close to the horizon is converted into ordinary particles, the whole mass of dark matter $\Delta m_{a}=M j / \eta$ needed to obtain the same current is much lower than the mass of the ordinary matter accreted onto the black hole, leading to its observed light radiation.

The source of the UHECR energy is the decay of superheavy particles at the GU energies into quarks and leptons which, according to our reasoning in Part 3 of our paper, led to the origination of the baryon charge of the Universe. The mass of a superheavy particle is converted into the energy of light particles whose flow can come from the black hole to the Earth similarly to photons. The black hole plays the role of a cosmic accelerator or supercollider creating the conditions for transforming the long-living component of $X$-particles into short living and its decay.

Now let us evaluate the density of dark matter needed to form the observed UHECR flow. Suppose that dark matter is uniformly distributed with a density typical for the ordinary matter in central parts of the galaxies, $\rho=10^{-20} \mathrm{~g} / \mathrm{cm}^{3}$. Let us take the typical velocities of dark matter particles at large distance from the central black hole as $v_{\infty} \approx 10^{8} \mathrm{~cm} / \mathrm{s}$ (these are stellar velocities in the central parts of galaxies). The capture cross-section for nonrelativistic particles by a Schwarzschild black hole is given by (see Eq. (3.9.1) in [9])

$$
\sigma_{c}=4 \pi\left(\frac{c}{v_{\infty}}\right)^{2} r_{g}^{2}
$$

Here $r_{g}$ is the horizon radius of the black hole.

The capture cross-section for a rotating black hole is of the same order $\left[9\right.$. Taking $M_{B H}=10^{8} M_{\odot}\left(M_{\odot}\right.$ is solar mass), one obtains for the velocity of the dark matter accretion onto the black hole $\Delta m_{a}=$ $\sigma_{c} v_{\infty} \rho \approx 3 \times 10^{28} \mathrm{~g} /$ year, which is consistent with our evaluation of the Auger observation $j \approx 10^{38}$ UHECR/year. This nontrivial coincidence can be an argument for our reasoning.

One must mention that conversion of dark matter into UHECR is effective only for objects with large quantity of the diffuse dark matter close to the black hole. This situation can occur only in AGN and is improbable for ordinary galaxies. From (11) one can see that capture of dark matter by a black hole is proportional to the square of black hole mass, so that the UHECR flow from a black hole of stellar masses is negligible.

We have no observation data for the distribution of dark matter in central regions of galaxies with AGN. If one takes for the distribution density of dark matter the numerical profiles

$$
\rho(r)=\frac{\rho_{0}}{\left(r / r_{0}\right)^{\beta}\left(1+r / r_{0}\right)^{3-\beta}}
$$

with $\beta=1$ for Navarro-Frenk-White profile [10, $\beta=1.5$ for Moore profile, $r_{0}=45 \mathrm{kpc}, \rho_{0}=$ $10^{-24} \mathrm{~g} / \mathrm{cm}^{3}$ [1], then one again obtains $\Delta m_{a} \sim$ $2 \times 10^{28}-10^{30} \mathrm{~g} /$ year.

So our estimate leads to a reasonable number for the accretion of supermassive dark matter particles onto a black hole. This dark matter can be considered to be a source of UHECR arising from the decay of supermassive particles into visible matter close to the horizon of a supermassive black hole. 
The maximum energy of the observable cosmic rays is not higher than $3 \times 10^{20} \mathrm{eV}$. Note that the absence of observations of cosmic rays with higher energy does not contradict the hypothesis that the source of UHECR is the decay of superheavy particles of the mass of the order $M=10^{14} \mathrm{GeV}$ near the horizon of the central black holes in AGN. The observable luminosity of AGN is $10^{43}-10^{48} \mathrm{erg} / \mathrm{s}$ close to the centre. In such a dense flow of radiation protons of ultrahigh energy will have many photonuclear collisions leading to large energy decrease (see, e.g., [12], ch. $4, \S 5$ ).

Now let us discuss the possible physical mechanism of conversion of dark matter into visible matter in AGN. It is reasonable to think that AGN, unlike other black holes, are rapidly rotating supermassive black holes with an angular momentum close to the critical value. Then one has the well-known Penrose mechanism [7. An incoming particle in the ergosphere decays into two or more particles, one with negative energy goes inside the black hole while other particles with the opposite momentum and an energy larger than the incoming one go to the ambient space.

A new feature of this process is that a long-living particle of dark matter can decay into a particle of larger mass which can be identified with the shortliving component $X_{s}$ decaying into particles of visible matter - quarks and leptons, as it had happened according to our scenario in the early Universe. If the energy transfer in such process is larger than the GU scale, then neither the baryon charge nor the conserved charge of dark matter particles are conserved. Note that similar processes of decay into a particle of larger mass than that of the initial one (forbidden in Minkowski space) could occur in the early expanding Universe [14, 15. CPT-invariance is broken in such processes as the consequence of breaking of $T$-invariance in a nonstatic metric.

Let us give some necessary formulas and estimates. The Kerr metric of a rotating black hole in the Boyer-Lindquist coordinates has the form

$$
\begin{aligned}
& d s^{2}=d t^{2}-\left(r^{2}+a^{2} \cos ^{2} \theta\right)\left(\frac{d r^{2}}{r^{2}-2 m r+a^{2}}+d \theta^{2}\right) \\
& -\left(r^{2}+a^{2}\right) \sin ^{2} \theta d \varphi^{2}-\frac{2 m r\left(d t-a \sin ^{2} \theta d \varphi\right)^{2}}{r^{2}+a^{2} \cos ^{2} \theta},
\end{aligned}
$$

where $m$ is the black hole mass, $a m$ is the angular momentum $(a<m)$. Here we use units $G=c=1$. At the event horizon

$$
r=r_{H} \equiv m+\sqrt{m^{2}-a^{2}} .
$$

The surface called the stationary limit surface is given by

$$
r=r_{0} \equiv m+\sqrt{m^{2}-a^{2} \cos ^{2} \theta} .
$$

The ergosphere is the region of space-time between the horizon and the stationary limit surface. Inside the ergosphere, the Killing vector of time translation $(1,0,0,0)$ becomes the spacelike, and the energy of a particle in this region measured by the observer in infinity can be negative. This leads to a possibility of the physical process of getting the energy from a rotating black hole. However there are some constraints on this energy. In case of particle decay, one has the Wald inequality (see, e.g., 13, $§ 65$ ).

If $E_{u}$ is the specific energy (energy/rest-mass) of a particle falling onto a black hole, $\varepsilon$ and $v$ are the specific energy and relative velocity of one of the fragments into which the particle decayed, then

$$
\gamma\left(E_{u}-|v| \sqrt{E_{u}^{2}-\left(1-\frac{2 m r}{r^{2}+a^{2} \cos ^{2} \theta}\right)}\right) \leq \varepsilon,
$$

where $\gamma=1 / \sqrt{1-|v|^{2}}$ and $r_{H}<r<r_{0}$.

Let us consider the initial particle falling onto the black hole to be nonrelativistic far from from the black hole, i.e. $E \approx 1$. If the initial particle of mass $M$ decays into two particles one of which is a light particle with mass $\mu$, then one can take $|v| \approx 1$ and $\gamma \approx M / 2 \mu$. So one gets a constraint on the additional energy (mass) of the other fragment:

$$
\Delta E \leq \frac{M}{2}\left(\sqrt{\frac{2 m r}{r^{2}+a^{2} \cos ^{2} \theta}}-1\right) .
$$

So one can obtain the following estimate of the additional energy (mass) obtained in the process of decay of the massive particle into light and heavier particles in the ergosphere of a black hole with the specific angular momentum $a$ :

$$
\Delta E \leq \frac{M}{2}\left[\left(1-\frac{1}{2}\left(1-\sqrt{1-\frac{a^{2}}{m^{2}}}\right)\right)^{-1 / 2}-1\right] .
$$

The maximal value $\Delta E_{M} \approx M(\sqrt{2}-1) / 2$ is achieves for a black hole with an angular momentum close to the critical value $a=m$. Full description of the process needs some $S$-matrix theory.

If the mass of short-living $X_{s}$-particles is larger than the mass of long living ones, then their creation in the Penrose process and consequently the appearance of the UHECR in decays of the short living component of $X$-particles will be obtained only for rapidly rotating black holes.

So the necessary condition for the Penrose mechanism - ultra relativistic movement of decay products as well as condition for converting of superheavy 
dark matter particles into quarks and leptons, i.e., large value of the relative energy-momentum in interaction of these particles, can be fulfilled in process under consideration. Outgoing particles with energy larger than the Grand Unification scale can collide with other superheavy particles and ordinary matter. As a result, macroscopic amount of dark matter can be "burnt" close to AGN and create UHECR. So AGN can work as a large cosmic collider.

\section{Superheavy particles in the early Universe}

The total number of massive particles created in the Friedmann radiation-dominated Universe (with the scale factor $a(t)=a_{0} t^{1 / 2}$ ) inside the horizon is, as it is known [5],

$$
N=n^{(s)}(t) a^{3}(t)=b^{(s)} M^{3 / 2} a_{0}^{3},
$$

where $b^{(0)} \approx 5.3 \times 10^{-4}$ for scalar and $b^{(1 / 2)} \approx 3.9 \times$ $10^{-3}$ for spinor particles. It occurs that $N \sim 10^{80}$ for $M \sim 10^{14} \mathrm{GeV}[6$. The radiation dominance at the end of inflation era for dark matter is important for our calculations. If it is dust-like, the results will be different (see further). However, this radiation is formed not by our visible particles. It is quintessence or some mirror light particles, not interacting with ordinary particles.

For the time $t \gg M^{-1}$, there is a transition era from a radiation-dominated model to a dust model of superheavy particles,

$$
t_{X} \approx\left(\frac{3}{64 \pi b^{(s)}}\right)^{2}\left(\frac{M_{P l}}{M}\right)^{4} \frac{1}{M},
$$

where $M_{P l} \approx 1,2 \times 10^{19} \mathrm{GeV}$ is the Planck mass. If $M \sim 10^{14} \mathrm{GeV}, t_{X} \sim 10^{-15} \mathrm{~s}$ for scalar and $t_{X} \sim$ $10^{-17} \mathrm{~s}$ for spinor particles.

Let us call $t_{X}$ the "early recombination era".

The formula for created particles in the volume $a^{3}(t)$ can be written as

$$
N(t)=\left(\frac{a\left(t_{C}\right)}{t_{C}}\right)^{3} b^{(s)}
$$

where $t_{C}=1 / M$ is the Compton time, $b^{(s)}$ depends on the form of $a(t)$. From (11) one can see the effect of connection of the number of created particles with the number of causally disconnected parts on the Friedmann Universe at the Compton time of its evolution.

For scale factor $a(t)=a_{0} t^{\alpha}$ from Eq. (11) it follows that $N=b^{(s)} M^{3(1-\alpha)} a_{0}^{3}$. Therefore for a dust-like end of inflation era one has $N \sim M$, and the ratio of the $X$-particles energy density $\varepsilon_{X}$ to the critical density $\varepsilon_{\text {crit }}$ is time-independent $\left(\varepsilon_{X}<\varepsilon_{\text {crit }}\right.$ for $M<M_{P l}$ ).

Let us define $d$, the permitted part of longliving $X$-particles, from the condition: at recombination time $t_{r e c}$ in the observable Universe one has $d \varepsilon_{X}\left(t_{r e c}\right)=\varepsilon_{\text {crit }}\left(t_{r e c}\right)$. It leads to

$$
d=\frac{3}{64 \pi b^{(s)}}\left(\frac{M_{P l}}{M}\right)^{2} \frac{1}{\sqrt{M t_{r e c}}} .
$$

For $M=10^{13}-10^{14} \mathrm{GeV}$ one has $d \approx 10^{-12}-10^{-14}$ for scalar and $d \approx 10^{-13}-10^{-15}$ for spinor particles. So the lifetime of the main part or all $X$-particles must be smaller or equal than $t_{X}$.

Earlier we constructed, by analogy with the $K^{0}$ meson theory, a model [3] which can give: (a) shortliving $X$-particles decay at the time $\tau_{s}<t_{X}$ (more wishful is $\tau_{s} \sim t_{C} \approx 10^{-38}-10^{-35}$ s, i.e., the Compton time for $X$-particles); (b) long-living particles decay with $\tau_{l}>1 / M$. Remaind that in order to have $m_{X_{l}}<m_{X_{s}}$ one must take $A>0$ in Eq. (7) of [2].

If $\tau_{l}$ is larger than the GU symmetry breaking time, it can happen that some quantum number similar to a baryon charge can be conserved, leading to some effective time $\tau_{l}^{\text {eff }}>t_{U} \approx 4.3 \times 10^{17} \mathrm{~s}$ ( $t_{U}$ is the age of the Universe). The small $d \sim 10^{-15}-10^{-12}$ part of long-living $X$-particles with $\tau_{l}>t_{U}$ forms the dark matter.

For $t_{l}^{\text {eff }} \leq 10^{27} \mathrm{~s}$ one could have the observable flow of UHECR from the decay in our Galaxy [16]. But in this case one must get a strong anisotropy in the direction to the center of the Galaxy [11]. However, Auger experiments do not show such an anisotropy, and one must suppose $t_{l}^{\text {eff }}>10^{27} \mathrm{~s}$.

In our papers 3 we considered observable entropy of the Universe production due to decay of of $X$ particles. To get the observable value of the entropy, it is sufficient to suppose the interaction of the long-living component of $X$-particles with the baryon charge (i.e. ordinary matter). Then if we introduce the effective Hamiltonian of such interaction with baryon matter as

$$
H^{d}=\left(\begin{array}{cc}
0 & 0 \\
0 & -i \gamma
\end{array}\right)
$$

one can write this parameter as being proportional to the concentration of particles: $\gamma=\alpha n^{(s)}(t)$. Then

$$
\alpha=\frac{-3 \ln d}{128 \pi\left(b^{(s)}\right)^{2}} \frac{M_{P l}^{2}}{M^{4}} .
$$

For $M=10^{14} \mathrm{GeV}$ and $d=10^{-14}$ one obtains $\alpha \approx$ $10^{-40} \mathrm{~cm}^{2}$. Thus such a mechanism of decay of the 
long-living component of $X$-particles was important in the early Universe at time $t_{X}$. In the modern era, due to smallness of the interaction cross-section and concentration of $X$-particles, this mechanism of decay does not give non-negligible contribution to the observable UHECR flow.

The observed entropy in this scenario originates due to transformation of $X$-particles into light particles: quarks, antiquarks and some particle similar to $\Lambda^{0}$ in $K^{0}$-meson theory, having the same quantum number as $X$. Baryon charge is created close to the time $t_{s}$, which can be equal to the Compton time of $X$-particles $t_{C} \sim 10^{-38}-10^{-35}$ s.

Our scheme can also work for spinor particles. Then it is possible to investigate some version of the see-saw mechanism [18] for Majorana neutrinos in GU theory, so that heavy sterile neutrinos form the dark matter.

\section{Acknowledgments}

This work was supported by the Ministry of Science and Education of Russia, grant RNP.2.1.1.6826.

\section{References}

[1] The Pierre Auger Collaboration, Science 318, 938 (2007).

[2] A. A. Grib and Yu. V. Pavlov, Mod. Phys. Lett. A 23, 1151 (2008).

[3] A. A. Grib and Yu. V. Pavlov, Int. J. Mod. Phys. D 11, 433 (2002); Int. J. Mod. Phys. A 17, 4435 (2002); Gravit. \& Cosmology 8 Suppl., 148 (2002); Gravit. \& Cosmology 12, 159 (2006).

[4] V. K. Dubrovich and M. Yu. Khlopov, JETP Lett. 77, 335 (2003).

[5] A. A. Grib, S. G. Mamayev, and V. M. Mostepanenko, Vacuum Quantum Effects in Strong Fields (Friedmann Lab. Publ., St. Petersburg, 1994).

[6] A. A. Grib and V. Yu. Dorofeev, Int. J. Mod. Phys. D 3, 731 (1994).

[7] R. Penrose, Rivista Nuovo Cimento I, Num. Spec., 252 (1969).

[8] K. Greisen, Phys. Rev. Lett. 16, 748 (1966); G. T. Zatsepin and V. A. Kuzmin, JETP Lett. 4, 78 (1966).

[9] Ya. B. Zel'dovich and I. D. Novikov, Gravitation Theory and Stars Evolution (Nauka, Moscow, 1971).
[10] J. F. Navarro, C. S. Frenk, and S. D. M. White, Astrophys. J. 462, 563 (1996).

[11] R. Aloisio, V. Berezinsky, and M. Kachelriess, Phys. Rev. D 74, 023516 (2006).

[12] Astrophysics of Cosmic Rays, Ed. by V. L. Ginzburg (Nauka, Moscow, 1990).

[13] S. Chandrasekhar, The Mathematical Theory of Black Holes (Oxford, Oxford Univ. Press, 1983).

[14] L. H. Ford, Nucl. Phys. B 204, 35 (1982).

[15] A. A. Grib and Yu. V. Kryukov, Yadernaya Fizika 48, 1842 (1988) [Engl. transl. in Sov. J. Nucl. Phys. (USA) 48, 1109 (1988)].

[16] V. Berezinsky, P. Blasi, and A. Vilenkin, Phys. Rev. D 58, 103515 (1998).

[17] H. V. Klapdor-Kleingrothaus and K. Zuber, Particle Astrophysics (Institute of Physics, Bristol, 1997).

[18] M. Gell-Mann, P. Ramond, and S. Slansky, in Supergravity, Ed. by P. van Niewenhuizen and D. Z. Freedman (Noth Holland, Amsterdam, 1979), pp. 315-321. 